\title{
STRATÉGIES D'UTILISATION DE L'HABITAT PAR LES ALEVINS POST-ÉMERGENTS DE TRUITE COMMUNE (SALMO TRUTTA) ET DE SAUMON ATLANTIQUE (SALMO SALAR).
}

\author{
P. GAUDIN (1) et M. HELAND (2), \\ avec la collaboration technique de J.C. VIGNES (2).
}

(1) URA CNRS 1974, Laboratoire d'Ecologie des Eaux Douces et des Grands Fleuves, Université Claude Bernard Lyon 1, 43 bd du 11 Novembre 1918, 69622 Villeurbanne Cedex, France.

(2) Laboratoire d'écologie des Poissons, INRA, Station d'Hydrobiologie de Saint-Pée-surNivelle, B.P. 3, 64310 Saint-Pée-sur-Nivelle, France.

\section{RÉSUMÉ}

Des observations réalisées en fluvarium pendant les deux semaines suivant l'émergence d'alevins de truite commune (Salmo trutta) et de saumon Atlantique (S. salar), montrent que ces deux espèces développent des stratégies différentes d'utilisation de l'habitat. Les alevins de saumon sont fortement inféodés au substrat, ils passent l'essentiel de leur temps au contact du fond et les tentatives de captures de proies et les comportements agressifs sont rares. Les alevins de truite occupent rapidement un poste de chasse où ils se tiennent en nage statique, bien détachés du substrat, les tentatives de captures de proies et les comportements agressifs sont beaucoup plus fréquents. Ces différences sont discutées en fonction de l'habitat sélectionné par les deux espèces à ce stade de développement.

\section{HABITAT USE STRATEGIES BY POST-EMERGENT FRY OF BROWN TROUT (SALMO TRUTTA) AND ATLANTIC SALMON (SALMO SALAR).}

\begin{abstract}
Observations performed in a flume during two weeks on post-emergent fry of brown trout (Salmo trutta) and Atlantic salmon ( $S$. salar) demonstrated that these two species used their habitat in different ways. Atlantic salmon were strongly substrate-bound : they spent most of their time lying on the bottom and prey capture attempts and agressive behaviour were scarce. Brown trout were precociously observed in static swimming position on a feeding station, facing the current away from the substrate ; prey capture attempts and agressive behaviour were much more frequent. These differences are discussed in relation with the fry habitat selection at this stage of their development.
\end{abstract}

\section{INTRODUCTION}

L'émergence est un événement capital dans le cycle vital des alevins de salmonidés. Ils abandonnent alors le milieu très protégé que constituent les graviers de la frayère et doivent rapidement apprendre à se déplacer et à se nourrir dans le courant tout en évitant les prédateurs (voir la revue présentée par HELAND et al., 1995). C'est sans doute compte-tenu des difficultés d'observation et d'expérimentation que peu de travaux se sont intéressés à l'utilisation de l'habitat pendant cette période, malgré son importance capitale pour la survie individuelle (voir cependant BEALL et al., 1989, 1994 ; BARDONNET, 1993 ; BARDONNET et GAUDIN, 1990 ; BARDONNET et HELAND, 1994). Par la suite, on constate que les alevins puis les juvéniles occupent des habitats qui correspondent tout à fait à leurs capacités à se déplacer et à se maintenir dans le courant. Ainsi, les alevins de saumon Atlantique (Salmo salar) vivent dans des radiers et rapides au courant assez vif 
(50 à $70 \mathrm{~cm} . \mathrm{s}^{-1}$ ) (HELAND et DUMAS, 1994), alors que les alevins de truite (S. trutta) vivent dans des zones où le courant est généralement inférieur à $30 \mathrm{~cm} \cdot \mathrm{s}^{-1}$ (HAURY et al., 1991). Chez ces deux espèces à la morphologie très voisine (particulièrement à ce stade), on ne sait cependant pas si ces aptitudes à utiliser des habitats différents sont effectives dès l'émergence ou si elles se mettent en place après une phase d'apprentissage plus ou moins longue. Les observations présentées ici portent sur les premiers jours de vie en eau libre de ces deux espèces, placées en conditions de milieu homogènes dans un fluvarium. Leur objectif est de déterminer expérimentalement si des aptitudes morphologiques et des comportements différents se manifestent dès l'émergence des alevins.

\section{MATÉRIEL ET MÉTHODES}

Les observations sont réalisées dans l'une des deux sections $(L \times I \times h=10 \mathrm{~m} \times 1 \mathrm{~m}$ $x 1 \mathrm{~m}$ ) du fluvarium de la station INRA de St Pée-sur-Nivelle (Pyrénées Atlantiques, France, (BARDONNET et HELAND, 1994)). 20 cages $(L \times I \times h=0,5 \mathrm{~m} \times 0,5 \mathrm{~m} \times 0,5 \mathrm{~m}$; vide de maille $=2 \mathrm{~mm}$ ) sont disposées tous les mètres, deux par deux, séparées par une paroi opaque, de manière à être traversées par l'ensemble de l'eau en mouvement. Les conditions physiques à l'intérieur des cages sont aussi homogènes que possible. Leur fond est recouvert d'une couche de graviers $(\approx 15 \mathrm{~cm})$ d'environ 10 à $50 \mathrm{~mm}$ de diamètre. La hauteur d'eau au milieu des cages varie entre 14 et $20 \mathrm{~cm}(16$ à $20 \mathrm{~cm}$ pour $90 \%$ des cages) et la vitesse du courant (moulinet OTT) varie de 12,4 à $20,1 \mathrm{~cm} . \mathrm{s}^{-1}$ en surface, de 11,1 à $18,9 \mathrm{~cm} \cdot \mathrm{s}^{-1}$ au milieu de la colonne d'eau et de 11,1 à $16,6 \mathrm{~cm} \cdot \mathrm{s}^{-1}$ à $1 \mathrm{~cm}$ du fond (pour $90 \%$ des mesures, respectivement $13-19,5,12,4-17,2$ et 12,4-16 cm.s. L'éclairement est de $2,5 \mathrm{~W} / \mathrm{m}^{2}(500 \pm 50 \mathrm{Lux})$ à la surface de l'eau. La photophase dure 13 heures, de $7 \mathrm{~h}$ à $20 \mathrm{~h}$ (heure solaire) et comprend deux phases de lumière atténuée (50 Lux) à l'aube et au crépuscule. La température de l'eau est de $12,5 \pm 1,5{ }^{\circ} \mathrm{C}$. Les observations de nuit sont réalisées grâce à une vidéo et un éclairage à infrarouge mobiles (Lampe Osram PAR 56, $300 \mathrm{~W}$ avec filtre IR, $\lambda>820 \mathrm{~nm}$ ).

Les alevins de truite (souche Ibarron, fécondation le 13/12/1990) et de saumon (souche Gave, fécondation le 3/12/1990) sont introduits par siphonnage sous les graviers le 11/02/1991 (environ une semaine après l'éclosion). Pour chaque espèce, trois densités sont introduites : un, deux et cinq individus par cage $\left(4,8\right.$ et $\left.20 / \mathrm{m}^{2}\right)$. Chaque densité est répétée trois fois et leur succession est tirée au hasard. Les observations débutent dès que le premier alevin émergent est observé et se déroulent du 1 au 13/03 pour les truites et du 4 au 18/03 pour les saumons. Les observations diurnes ont lieu le matin entre $9 \mathrm{~h}$ et $11 \mathrm{~h} 30$ et l'après-midi entre $16 \mathrm{~h}$ et $18 \mathrm{~h} 30$. Chaque cage est observée cinq minutes au cours desquelles sont notés : le nombre d'alevins émergés et, pour chaque alevin, le nombre de tentatives de capture (il est impossible de savoir si les proies sont capturées), le nombre de séquences agressives et une estimation (à $10 \%$ près) du temps consacré à quatre types d'activités (partiellement enfoui - posé sur la face ventrale et immobile - posé sur le substrat et érigé sur les nageoires - en nage statique face au courant détaché du substrat). Les observations de nuit ont lieu au moins une heure après le coucher du soleil. Chaque cage est observée environ deux minutes au cours desquelles sont notés : le nombre d'alevins présentant au moins une séquence de nage statique face au courant, les comportements agressifs et de capture et les autres déplacements. Le 19/03/91, les alevins sont pêchés, pesés et mesurés.

Afin que des proies soient disponibles en permanence pendant toute la durée de l'expérience, le contenu d'un surber prélevé dans la Nivelle est déversé tous les un à trois jours dans le fluvarium dès l'introduction des alevins. A partir de la première émergence, ces déversements sont toujours réalisés après une des deux séances d'observations diurnes.

Pour la présentation des résultats, les alevins sont répartis en 3 catégories pour l'analyse des effets de la densité ( 1,2 et $\geq 3$ individus par cage) et, pour tenir compte des effets de l'âge, trois périodes successives sont considérées pour chaque espèce (pour les truites, âge $1:$ du 1 au 5 , âge $2:$ du 6 au 9 et âge $3:$ du 10 au $13 / 03$ et pour les saumons, âge 1 : du 4 au 8 , âge 2 : du 9 au 13 et âge $3:$ du 14 au 17/03). 


\section{RÉSULTATS}

\section{Emergence et croissance des alevins}

L'émergence des truites s'est déroulée du 1 au 5/03 (survie $71 \%$ ) et celle des saumons du 4 au 13/03 (survie 100\%). La comparaison des tailles et poids moyens en fin d'expérience pour les deux espèces et pour deux groupes de densité ( $\leq 2$ et $\geq 3$ individus par cage) permet de mettre en évidence un effet significatif de l'espèce sur la longueur et le poids (test $t ; p<0,03$ ) et de la densité sur le poids des saumons (test $t ; p=0,02$ ). Les truites, qui sont un peu plus âgées que les saumons en fin d'expérience, sont en moyenne plus longues et plus lourdes que ces derniers : la longueur moyenne est de $33,3 \mathrm{~mm}$ pour les truites $(N=17, \sigma=2,3, \min -\max : 29-37 \mathrm{~mm})$ contre $31,2 \mathrm{~mm}$ pour les saumons $(N=24$, $\sigma=1,8$, min-max : $27-34 \mathrm{~mm})$ et le poids moyen est de $0,33 \mathrm{~g}$ pour les truites $(\sigma=0,07$, min-max : $0,19-0,44 \mathrm{~g})$ et seulement de $0,23 \mathrm{~g}$ pour les saumons $(\sigma=0,05$, min-max : $0,12-0,33 \mathrm{~g}$ ). A longueur égale, les saumons sont plus lourds dans les faibles densités que dans les fortes densités : le poids moyen est respectivement de $0,26 \mathrm{~g}(\mathrm{~N}=9, \sigma=0,05$, $\min -\max : 0,2-0,33 \mathrm{~g})$ contre $0,21 \mathrm{~g}(\mathrm{~N}=15, \sigma=0,05, \min -\max : 0,12-0,29 \mathrm{~g})$.

\section{Activité diurne}

Chez la truite, la proportion du temps que les alevins consacrent à la "nage face au courant" atteint $50 \%$ dès le 6ème jour après la première émergence (donc dès la fin de la période d'émergence) (Fig. 1). Cette activité de nage est associée à une proportion régulière d'activité : "posé, érigé sur les nageoires"(environ $20 \%$ ). Dès le 9ème jour, ces deux items représentent la totalité de l'activité des alevins, la "nage face au courant" se stabilisant entre 80 et $90 \%$. Chez le saumon, l'immobilité ("semi-enfouis" ou "posés sur la face ventrale") représente $50 \%$ de l'activité jusqu'au 6ème jour suivant la lère émergence (Fig. 1). La position "posé, érigé sur les nageoires" devient ensuite l'activité dominante (plus de $50 \%$ à partir du 9ème jour, correspondant à la fin de l'émergence). La nage face au courant ne s'intensifie que très lentement et ne dépasse $30 \%$ du temps qu'à la fin de la période d'observation.

Les comportements agressifs sont quasi absents chez le saumon (1/313 observations à la densité $\geq 3$ et $0 / 152$ à la densité 2$)$ et plus fréquents chez la truite (34/261 observations aux densités $\geq 2$ ) (Tab. I). Chez cette dernière espèce, l'effet de l'âge des alevins n'est pas significatif $\left(\chi^{2}=2,8 ; \mathrm{ddl}=1 ; p=0,095\right)$ mais celui de la densité est très net, la fréquence des agressions étant multipliée par environ 10 lorsqu'on passe de 2 à $\geq 3$ alevins par cage.

Les comportements alimentaires sont peu fréquents chez le saumon puisqu'on n'observe en moyenne que 0,12 tentatives de captures de proies par individu et par 5 minutes (Tableau I), soit moins de 20 par jour $(=18,9$, si on admet que ces tentatives se répartissent de manière homogène au cours des 13 heures de photophase). L'augmentation de la densité n'a pas d'effet sur la fréquence des tentatives de captures $\left(\chi^{2}=5,4 ; \mathrm{ddl}=2\right.$; $p=0,067$, non significatif) contrairement aux variations observées avec l'âge $\left(\chi^{2}=30,22\right.$; $\mathrm{ddl}=2 ; p=0,0001)$, mais la diminution observée au 2ème âge est inexpliquée. Chez la truite, les comportements alimentaires sont environ 20 fois plus fréquents que chez le saumon, soit près de 400 tentatives de capture par jour $(390,7$, si on se livre à la même estimation que cidesssus) (Tab. I). La fréquence des captures augmente fortement avec l'âge $\left(\chi^{2}=158,7\right.$; $d d l=2 ; p=0,0001)$ et la densité des alevins $\left(\chi^{2}=114,33 ; d d l=2 ; p=0,0001\right)$.

\section{Activité nocturne}

Parmi les divers types de déplacements observés, seuls les comportements de nage statique sont en nombre suffisant pour être considérés séparément. Les autres déplacements observés sont : des déplacements lents au contact ou près du fond, des nages contre le courant en pleine eau parfois au contact de la surface, des montées très rapides suivies d'une phase de nage désordonnée au contact de la surface et des allerretour très rapides en direction de la surface (avec ou sans contact avec la surface), parfois suivis de réenfouissements sous les graviers. Ces déplacements sont regroupés sous la même rubrique (Tab. II). Aucun comportement de capture n'a été observé. 

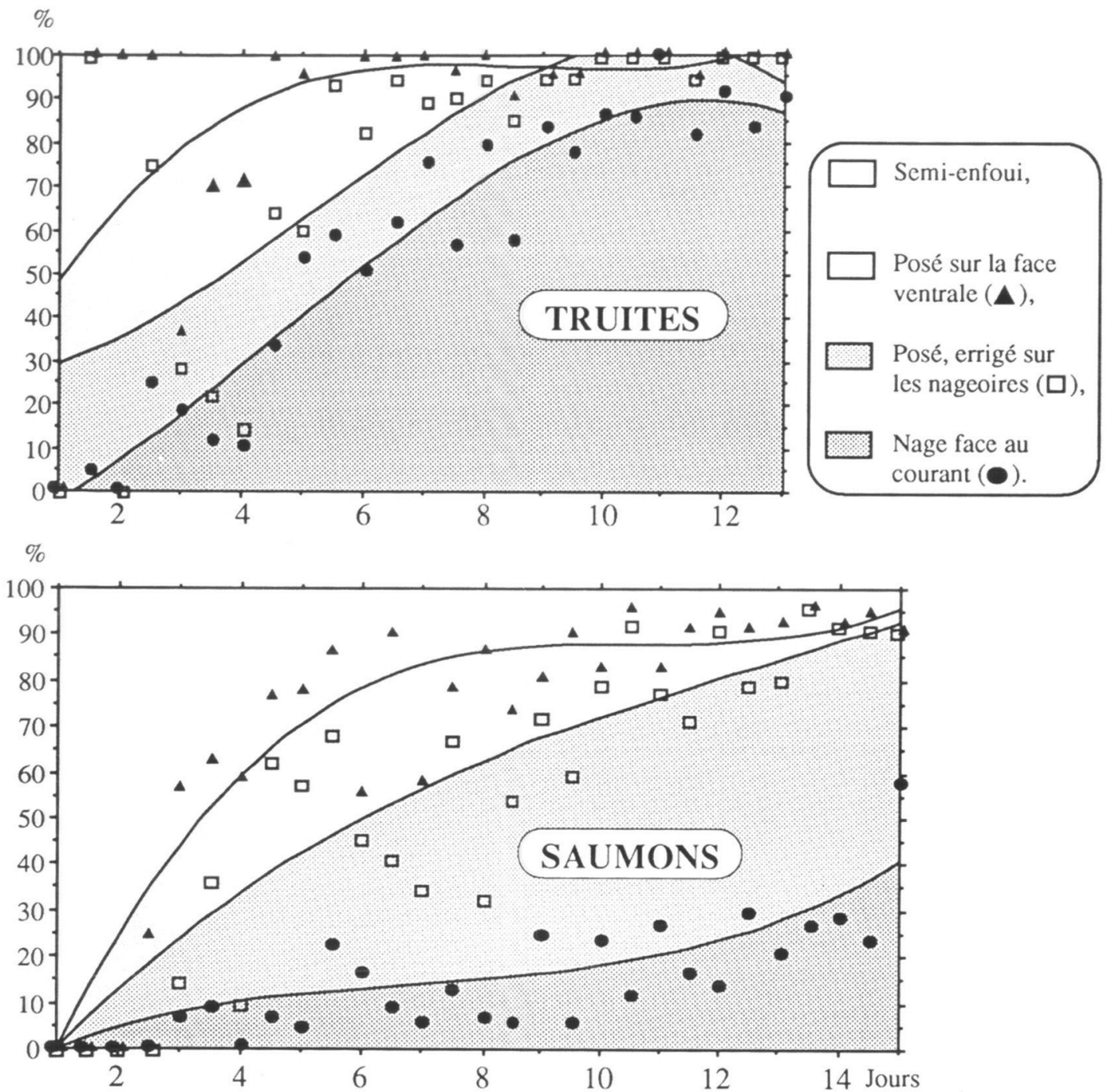

Figure 1 : Evolution du \% du temps consacré pendant la journée à chaque type d'activité par les alevins des deux espèces. Pour chacune des deux sessions quotidiennes d'observation, toutes les observations individuelles sont regroupées pour évaluer un \% global pour chaque type d'activité. Les courbes présentées à titre indicatif correspondent à des régressions polynomiales d'ordre 3 (dont les coefficients de régression sont tous significatifs au seuil $p<0,02$ ).

Figure 1 : Evolution of the \% time spent by day in each activity by fry of the two species. In each of the two daily observation sessions, all individual observations are pooled to assess a global \% for each activity. The indicative curves are third order polynomial regressions (the regression coefficients are significant at the level $p>0,02$ ).

Chez le saumon, la fréquence des déplacements est extrêmement faible (11\% pour l'ensemble des comportements, Tab. II) et ne varie pas de façon significative avec l'âge ou la densité. Chez la truite, on observe plus de déplacements ( $31 \%$ pour l'ensemble) mais également peu de variations avec l'âge et la densité. Seule l'augmentation de la fréquence des comportements de nage statique avec l'âge est significative (de 7 et $9 \%$ pour les deux premières classes d'âge à $25 \%$ pour la troisième, $\chi^{2}=7,353 ; d d l=2 ; p=0,023$ ). 
Tableau I : Effet de l'âge des alevins (voir texte) et de leur densité (en nombre d'alevins par cage) sur la fréquence des tentatives de capture ("captures") et des comportements agressifs ("agressions") par tranche de cinq minutes (fréquences par alevin entre parenthèses) au cours des observations réalisées de jour. La colonne "observations" correspond au nombre total d'observations d'alevins pour une espèce donnée pour la tranche d'âge ou la densité considérée.

Table I : Effect of fry age (see text) and density (in number of fry per cage) on the frequency of foraging attempts ("captures") and agressive behaviour ("agressions") by five minutes time period (frequency for each fry in brackets) in daily observations. Column "observations" indicates the total number of fry observations in each category (of species, age or density).

\begin{tabular}{|c|c|c|c|c|c|c|c|}
\hline & & \multicolumn{3}{|c|}{ TRUITES } & \multicolumn{3}{|c|}{ SAUMONS } \\
\hline & & Observations & Captures & Agressions & Observations & Captures & Agressions \\
\hline TOTAL & & 339 & $849(2,5)$ & $34(0,1)$ & 537 & $65(0,12)$ & $1(0)$ \\
\hline \multirow[t]{3}{*}{$\widehat{A} G E$} & 1 & 79 & $17(0,22)$ & 0 & 81 & $12(0,15)$ & 0 \\
\hline & 2 & 139 & $343(2,47)$ & $13(0,09)$ & 216 & $4(0,02)$ & 1 \\
\hline & 3 & 121 & $489(4,04)$ & $21(0,17)$ & 240 & $49(0,2)$ & 0 \\
\hline \multirow[t]{3}{*}{ DENSITE } & 1 & 78 & $36(0,46)$ & - & 72 & $3(0,04)$ & - \\
\hline & 2 & 94 & $186(1,98)$ & $2(0,02)$ & 152 & $16(0,11)$ & 0 \\
\hline & $\geq 3$ & 167 & $627(3,75)$ & $32(0,19)$ & 313 & $46(0,15)$ & 1 \\
\hline
\end{tabular}

\section{DISCUSSION}

Dans des conditions de milieu homogènes, nos résultats permettent d'établir deux modèles d'utilisation de l'habitat bien différents pour chacune des espèces pendant cette phase de post-émergence. De jour, la truite se détache du substrat dans les quelques jours qui suivent l'émergence pour adopter un comportement de nage intermittente ou de nage statique continue (HELAND, 1978). Dès que cette activité est établie, les captures deviennent fréquentes et les comportements agressifs courants, en particulier lorsque la densité est élevée (ici, au dessus de 8 individus par $\mathrm{m}^{2}$ ). De toute évidence, le saumon est beaucoup plus inféodé au substrat. II adopte dès l'émergence une position d'attente au contact du fond qui reste la principale de ses activités après 15 jours de vie en eau libre. Les tentatives de capture sont peu fréquentes et les comportements agressifs très rares. De nuit, l'ensemble de l'activité est faible, mais les différences observées entre les deux espèces vont dans le même sens: les truites sont plus actives que les saumons et commencent à présenter quelques comportements de nage face au courant à la fin de la période d'observation alors que l'activité des saumons n'évolue pas.

La tactique développée par les post-émergents de ces deux espèces correspond aux habitats sélectionnés dans le milieu naturel. Les alevins de saumons occupent des radiers où il serait trop coûteux en énergie, voire impossible pour ces jeunes stades incapables de résister à des vitesses de courant supérieures à $25 \mathrm{~cm} . \mathrm{s}^{-1}$ (HEGGENES et TRAAEN, 1988), d'attendre les proies en nage statique. Ils se tiennent donc immobiles, plaqués au contact du fond grâce à leurs nageoires pectorales qui agissent comme des déflecteurs (ARNOLD et al., 1991). Ils ne se déplacent que très rarement mais certainement très efficacement : le nombre de tentatives de captures observé ici est de l'ordre de grandeur de la ration quotidienne à laquelle on peut s'attendre chez des alevins de cette taille (quelques proies à quelques dizaines de proies selon leur taille, résultats non publiés). Cette 
Tableau II : Effet de l'âge des alevins (voir texte) et de leur densité (en nombre d'alevins par cage) sur la fréquence d'apparition des séquences ("Nage statique") et ("autres déplacements") durant la nuit (fréquences par alevin entre parenthèses). La colonne "Nombre observable" correspond aux effectifs d'alevins d'une espèce donnée observables le lendemain matin des observations.

Table II : Effect of fry age (see text) and density (in number of fry per cage) on the frequency of nightly observations of fry in static swimming ("Nage statique") and other displacements ("autres déplacements") (frequency for each fry in brackets). Column "Nombre observable" indicates the number of fry of each species observable the next morning.

\begin{tabular}{|c|c|c|c|c|c|c|c|}
\hline & & & TRUITES & & & AUMON & \\
\hline & & $\begin{array}{c}\text { Nombre } \\
\text { observable }\end{array}$ & $\begin{array}{c}\text { Nage } \\
\text { statique }\end{array}$ & $\begin{array}{c}\text { Autres } \\
\text { déplace- } \\
\text { ments }\end{array}$ & $\begin{array}{c}\text { Nombre } \\
\text { observable }\end{array}$ & $\begin{array}{c}\text { Nage } \\
\text { statique }\end{array}$ & $\begin{array}{c}\text { Autres } \\
\text { déplace- } \\
\text { ments } \\
\end{array}$ \\
\hline TOTAL & & 185 & $26(0,14)$ & $31(0,17)$ & 179 & $5(0,03)$ & $15(0,08)$ \\
\hline$\widehat{A} G E$ & 1 & 46 & $3(0,07)$ & $7(0,15)$ & 29 & $1(0,03)$ & $2(0,07)$ \\
\hline & 2 & 70 & $6(0,09)$ & $11(0,16)$ & 79 & $1(0,01)$ & $6(0,08)$ \\
\hline & 3 & 69 & $17(0,25)$ & $13(0,19)$ & 71 & $3(0,04)$ & $7(0,01)$ \\
\hline DENSITE & 1 & 42 & $9(0,21)$ & $5(0,12)$ & 24 & 0 & $1(0,04)$ \\
\hline & 2 & 44 & $5(0,11)$ & $13(0,3)$ & 54 & $1(0,02)$ & $5(0,09)$ \\
\hline & $\geq 3$ & 99 & $12(0,12)$ & $13(0,13)$ & 101 & $4(0,04)$ & $9(0,09)$ \\
\hline
\end{tabular}

discrétion va de pair avec une quasi-absence d'interactions agressives qui contribue de toute évidence à limiter aussi les dépenses d'énergie. Au contraire, les alevins postémergents de truite vivent dans des zones où le courant est moins vif et leur comportement est beaucoup moins économe sur le plan énergétique. Leur recherche des proies est beaucoup plus active et ils réalisent beaucoup de tentatives de capture infructueuses. Le chiffre moyen de 400 tentatives par jour reflète très imparfaitement la réalité dans la mesure où il dépasse 600/jour pour les individus les plus âgés et où il atteint presque ce niveau pour les fortes densités (585/jour), alors qu'il n'excède pas 70/jour pour les alevins isolés et ce quel que soit leur âge. Dans les fortes densités, cette débauche de tentatives de captures va de pair avec de fréquentes interactions agressives, coûteuses elles aussi en énergie. II est cependant improbable que les besoins énergétiques supplémentaires liés à la présence de congénères soient seuls responsables de la très forte augmentation de la fréquence des tentatives de captures lorsque les individus ne sont pas isolés. On peut penser que ces fréquents déplacements en direction de particules dérivantes participent également aux relations sociales, dans la mesure où ils permettent à certains individus de manifester leur dominance.

En tout état de cause, il est clair que, dès les premières journées de vie en eau libre, les alevins post-émergents adoptent d'emblée des comportements nettement différents même s'ils sont élevés dans un milieu aux caractéristiques morphodynamiques homogènes. Ces comportements correspondent à l'utilisation des habitats dans lesquels on rencontre les alevins puis les juvéniles dans le milieu naturel et on peut supposer qu'ils sont liés à des aptitudes différentes dès l'émergence, comme l'observent CRISP et HURLEY (1991). Cependant, dans le présent travail où les alevins des deux espèces vivent dans la même eau, on ne peut pas exclure que, malgré l'isolement visuel, une interaction de type olfactif ait pu s'établir et induire un comportement particulier de la part de l'une ou de l'autre des espèces. II était cependant indispensable, dans un premier temps, de confronter les 
deux espèces à des conditions parfaitement homogènes en n'utilisant qu'un seul et même milieu. On peut également noter que les comportements observés concordent parfaitement avec ce qu'on observe chez les stades plus avancés (HELAND, 1991 ; HELAND et DUMAS, 1994), ce qui contribue fortement à valider ces résultats.

\section{REMERCIEMENTS}

Ce travail, effectué dans le cadre du P.I.R. Environnement (1992-1995): "Modélisation de l'utilisation spatio-temporelle de l'habitat par les poissons", a été réalisé grâce à l'aide du personnel technique de la Station d'Hydrobiologie de Saint-Pée-surNivelle : S. GLISE, J. P. LANDRET, J.P. MARTINET, J. J. YANCI.

\section{BIBLIOGRAPHIE}

ARNOLD G.P., WEBB P.W., HOLFORD B.H., 1991. The role of the pectoral fins in station holding of Atlantic salmon parr (Salmo salar L.). J. Exp. Biol., 156, 625-629.

BARDONNET A., 1993. Use of visual landmarks by young trout (Salmo trutta) during their diel downstream post-emergence displacement in experimental channels. J. Fish Biol., 43, 375-384.

BARDONNET A., GAUDIN P., 1990. Diel pattern of downstream post-emergence displacement of grayling (Thymallus thymallus L., 1758). J. Fish Biol., 37, 623-627.

BARDONNET A., HELAND M., 1994. The influence of potential predators on the habitat preferenda of emerging brown trout. J. Fish Biol., 45 (Supplement A), 131-142.

BEALL E., HELAND M., MARTY C., 1989. Interspecific relationships between emerging Atlantic salmon, Salmo salar, and coho salmon, Oncorhynchus kisutch, juveniles. J. Fish Biol., 35 (Supplement A), 285-293.

BEALL E., DUMAS J., CLAIREAUX D., BARRIERE L., MARTY C., 1994. Dispersal patterns and survival of Atlantic salmon (Salmo salar) juveniles in a nursery stream. ICES J. Mar. Sci., 51, 1-9.

CRISP D.T., HURLEY M.A, 1991. Stream channel experiments on downstream movement of recently emerged trout, Salmo trutta L., and salmon, S. salar L. - I. Effect of four different water velocity treatments upon dispersal rate. J. Fish Biol., 39, 347-361.

HAURY J., OMBREDANE D., BAGLINIERE J.L., 1991. L'habitat de la truite commune (Salmo trutta L.) en cours d'eau. In BAGLINIERE J.L., MAISSE G., eds., La truite, biologie et écologie, 47-96, INRA, Paris.

HEGGENES I., TRAAEN T., 1988. Downstream migration and critical water velocities in stream channels for fry of four salmonid species. J. Fish Biol., 32, 717-727.

HELAND M., 1978. Observations sur l'établissement du comportement de nage face au courant chez l'alevin de truite, Salmo trutta L., en ruisseau artificiel. Ann. Limnol., 14, 273-280.

HELAND M., 1991. Organisation sociale et territorialité chez la truite commune immature au cours de l'ontogenèse. In BAGLINIERE J.L., MAISSE G., eds., La truite, biologie et écologie, 47-96, INRA, Paris.

HELAND M., DUMAS J., 1994. Ecologie et comportement des juvéniles. In GUEGUEN C., PROUZET P., eds., Le saumon Atlantique : biologie et gestion de la ressource, 29-46, IFREMER, Brest.

HELAND M., GAUDIN P., BARDONNET A., 1995. Mise en place des premiers comportements et utilisation de l'habitat après l'émergence chez les salmonidés d'eau courante. Bull. Fr. Pêche Piscic., 337/338/339. 